\title{
Numerical simulation of electromechanical activity of the gastric smooth muscle
}

\author{
O. A. Al-Qabandi \& R. N. Miftahof \\ Arabian Gulf University, Kingdom of Bahrain
}

\begin{abstract}
A null-dimensional mathematical model of the myoelectrical activity of the gastric smooth muscle (myofiber) is studied numerically. Based on real morphological and electrophysiological data, the model assumes that: the kinetics of $\mathrm{L}$ - and T-type $\mathrm{Ca}^{2+}, \mathrm{Ca}^{2+}$-activated $\mathrm{K}^{+}$, voltage dependent $\mathrm{K}^{+}$and $\mathrm{Cl}^{-}$ channels determines the electrical activity of the myofiber; the enteric nervous system is represented by the primary sensory and motor neurons; the pacemaker input is provided by the interstitial cells of Cajal through receptor linked L-type $\mathrm{Ca}^{2+}$ channels located on the smooth muscle membrane. The dynamics of propagation of the wave of depolarization along the unmyelinated nerve axons satisfy the Hodgkin-Huxley model; the electrical activity of the neural soma reflects the interaction of $\mathrm{N}$-type $\mathrm{Ca}^{2+}$ channels, $\mathrm{Ca}^{2+}$-activated $\mathrm{K}^{+}$and voltage dependent $\mathrm{Na}^{+}, \mathrm{K}^{+}$and $\mathrm{Cl}^{-}$channels; and the smooth muscle is modeled as a nulldimensional contractile system which dynamics is determined entirely by the concentration of cytosolic calcium. The model reproduces: the mechanical excitation of the free nerve endings of the mechanoreceptor of the receptive field of the pathway; the electrical processes of the propagation of excitation along the afferent and efferent neural circuits; the chemical mechanisms of nerve-pulse transmission at the synaptic zones; the slow wave and bursting type electrical activity; cytosolic calcium concentration; the dynamics of active force generation. Results of numerical simulations of mechanical stimulation of the gastric myofiber have revealed different mechanical responses. Thus, with increased frequency and intensity of excitation there is a shift from phasic to tonic bursting and the development of long-lasting contractions. Comparison of theoretical and experimental results has revealed good qualitative and quantitative agreement.
\end{abstract}

Keywords: numerical model, gastric myofiber, electromechanical activity. 


\section{Introduction}

The gastric smooth muscle is embedded into a network of collagenous and elastin fibers that are coupled via gap junctions into distinct muscle layers. Their mechanical activity - contractions and relaxations - is responsible for grinding, mixing, and releasing chyme from the stomach into the duodenum. Contractions are the outcome of a sequence of complex electromechanical events that involve the myenteric nervous plexus, endocrine and paracrine regulatory mechanisms and intracellular processes in the muscle tissue per se. It is generally accepted that a pacemaker role in the gastrointestinal tract is attributed to interstitial cells of Cajal (ICC). They have been histologically identified as cells located in between the circular and longitudinal muscle layers [1, 2]. Electrophysiological studies have revealed that ICCs are connected via cholinergic synapses with Ltype $\mathrm{Ca}^{2+}$ channels on the smooth muscle membrane and impart a chronotropic effect of their conductivity. Additionally, balanced activity of the T-type $\mathrm{Ca}^{2+}$ channels, $\mathrm{Ca}^{2+}$-activated $\mathrm{K}^{+}$, voltage-dependent $\mathrm{K}^{+}$, and leak $\mathrm{Cl}^{-}$channels, is responsible for a broad repertoire of recorded electrical phenomena including slow waves and spikes.

To study the gastric smooth muscle in vivo and in vitro has been a challenging task with a limited success. Although experimental findings have revealed basic electrophysiological patterns of activity that are similar to the ones observed in other parts of the gastrointestinal tract, the exact response of the gastric muscle greatly depends on its actual physiological state. To control these states is an exigent unsolved experimental problem. Recently, a number of mathematical, in silico, models have been proposed to simulate the gastric smooth muscle under normal and pathological conditions [3-7]. However, most of these models suffer from biological insufficiencies and thus have limited clinical applications.

The aim of this work is to analyze the dynamics of electromechanical processes in a gastric myofiber in response to mechanical stimuli.

\section{Model outline}

A mathematical model of the gastric smooth muscle and its foundations are given elsewhere, and the reader is advised to consult our previous publications for full details $[8,9]$. The model assumes that:

i) the gastric smooth muscle (myofiber) is embedded in the extracellular matrix of collagen and elastin fibers; the myofiber has nonlinear viscoelastic characteristics; inactive smooth muscle cells, collagen, elastin fibers of the extracellular matrix define the "passive", and intracellular contractile proteins describe the "active” component, of its total force;

ii) contractions of the myofiber are considered to be isometric and deformations are finite;

iii) smooth muscle cells possess properties of an electromechanical biological continuum; additionally, they possess cable and myogenic electrical properties; myogenic electrical events are a result of activity 
of the fast (T-type) and slow (L-type) inward $\mathrm{Ca}^{2+}, \mathrm{BK}_{\mathrm{Ca}}$, voltage dependent $\mathrm{K}_{\mathrm{v}}^{+}$and leak $\mathrm{Cl}^{-}$currents; the muscle is in the silent state and the transformation to a firing state is a result of depolarization and/or stretch deformation that alters the conductance for L- and/or T-type $\mathrm{Ca}^{2+}$ channels;

iv) a neuronal circuit contain the sensory and motor neurons linked via cholinergic synapses; the dynamics of the action potential generation and propagation within the neuronal elements satisfy the HodgkinHuxley formalism; electrochemical coupling in the synapse is adequately described by the Michaelis-Menten kinetics.

The system of nonlinear partial and ordinary differential equations has been solved numerically using ABS Technologies ${ }^{\circledR}$ computational platform.

\section{Results}

\subsection{Response to a single stretch}

Excitation of the myofiber by the deformation of mechanoreceptors in the afferent pathway involves the following sequence of events: i) deformation of the free nerve endings of the mechanoreceptors; ii) action potential propagation along the unmyelinated fiber of the primary sensory neuron; iii) action potential generation at the soma of the primary sensory neuron and propagation of the wave of depolarization along the nerve axon towards the secondary sensory neuron; iv) electrochemical coupling (acetylcholine release, diffusion and binding with choline receptors) at the axo-dendritic synapse and generation of the EPSP; v) generation of the action potential at the soma of the secondary sensory neuron and its propagation along the nerve axon towards the cholinergic synapse located on the smooth muscle membrane; vi) initiation of chemical mechanisms of acetylcholine release and the generation of an excitatory postsynaptic potential; vii) activation of slow L-type $\mathrm{Ca}^{2+}$ channels and depolarization of the smooth muscle membrane; viii) active force generation as a result of an increase in intracellular $\left[\mathrm{Ca}^{2+}\right]$; ix) calculation of the deformation in terms of the force being generated, which leads to excitation of the free nerve endings of the mechanoreceptors.

A single stretch of the myofiber of the intensity $\varepsilon=0.1\left(\varepsilon=\left(l-l_{0}\right) / l_{0}\right)$ and duration $2 \mathrm{~s}$ is not enough to alter the receptor potential. A gradual increase in the level of the deformation leads to an increase in the amplitude of the electrical response. For $\varepsilon=0.85$ applied over $1 \mathrm{~s}$, the deformation evokes a single receptor potential at the dendrites of the mechanoreceptors of maximum amplitude $82.5 \mathrm{mV}$.

The dendritic potential causes the development of an action potential with an amplitude of $69 \mathrm{mV}$. It propagates along the unmyelinated axon of the primary sensory neuron at a constant velocity, $17.5 \mathrm{~cm} / \mathrm{s}$. On the soma of the primary neuron it initiates an excitatory membrane current with the generation of a single action potential of the average amplitude, $81.3 \mathrm{mV}$. The pattern of discharges 
resembles a bursting chaos type of electrical activity with the frequency 8$10 \mathrm{~Hz}$.

The action potential elicited at the soma of the primary sensory neuron propagates along the unmyelinated axon and reaches the axo-somatic synapse. Here the depolarization of the presynaptic membrane activates a short-term influx of calcium into the terminal. The concentration of $\mathrm{Ca}^{2+}$ quickly rises to a maximum of $19.4 \mathrm{mM}$. Some of the ions are immediately absorbed by the buffer system while others diffuse towards the vesicles. They bind with the active centers and initiate the release of acetylcholine (ACh). The rate of release is not constant but depends on the concentration of cytosolic $\mathrm{Ca}^{2+}$. Thus, at the beginning of the process, the free fraction increases at a rate of $0.5 \mathrm{mM} / \mathrm{ms}$ and reaches a maximum of $1.6 \mathrm{mM} / \mathrm{ms}$. During the whole cycle about $10 \%$ of vesicular ACh is released.

With the achievement of a free ACh concentration of $10.6 \mathrm{mM}$, neurotransmitter release into the synaptic cleft begins. According to the calculated data, $5.38 \mathrm{mM}$ equivalent to $50 \%$ of free $\mathrm{ACh}$, diffuses from the presynaptic terminal. The main part of ACh reaches the postsynaptic membrane and reacts with the choline receptors on the soma of the secondary sensory neuron. This leads to the generation of the fast excitatory postsynaptic potential. It rises as a step function achieves its maximum, $87.1 \mathrm{mV}$.

This level of EPSP is sufficient to excite the secondary sensory neuron. It discharges a single train of pulses of amplitude $102.1 \mathrm{mV}$ at a constant frequency $10 \mathrm{~Hz}$. The observed pattern resembles a regular bursting mode of electrical activity. Both the dynamics of propagation of the wave of excitation along the unmyelinated axon of the secondary sensory neuron and the dynamics of electrochemical coupling at the L-type $\mathrm{Ca}^{2+}$ channels on the smooth muscle membrane are essentially the same (quantitatively and qualitatively) as the dynamics of the electrical processes described above. The generated EPSP of an amplitude $87.1 \mathrm{mV}$ exceeds the threshold value for the activation of the permeability of L-type calcium channels on the smooth muscle membrane. This causes cyclic transitory changes in the observed myoelectrical pattern: (i) slow wave mode transforms to bursting chaos, (ii) bursting chaos transforms to regular bursting with generation of spikes on the crests of slow waves, (iii) regular bursting converts back to bursting chaos and slow wave mode (fig. 1). Thus, in the beginning the system displays periodic activity with the generation of waves of depolarization of the amplitude $31 \mathrm{mV}$ and frequency $0.13 \mathrm{~Hz}$. The wave of depolarization achieves its maximum in $1.5 \mathrm{~s}$, followed by a plateau of $2.9 \mathrm{~s}$ and finally slowly decreases to the resting value, $-61.8 \mathrm{mV}$. At the plateau there are spikes of variable amplitude ranging from 16 to $3 \mathrm{mV}$ that occur at an average frequency of $5.6 \mathrm{~Hz}$. Later the regular slow wave pattern changes to bursting chaos. The frequency of slow waves decreases, $0.08 \mathrm{~Hz}$, while the amplitude remains unchanged. Fast action potentials are generated at the top of waves at a frequency of $3.7 \mathrm{~Hz}$. Their firing level is low at the beginning and increases towards the end of the burst. Later on the myofiber displays repetitive regular bursting. The burst amplitude, burst duration and number of spikes per burst all increase. The action potentials have a maximum 
amplitude of $75 \mathrm{mV}$ and oscillate at a maximum frequency of $3.5 \mathrm{~Hz}$. With the decrease in activation of channels the system transforms to a short term chaotic bursts mode.
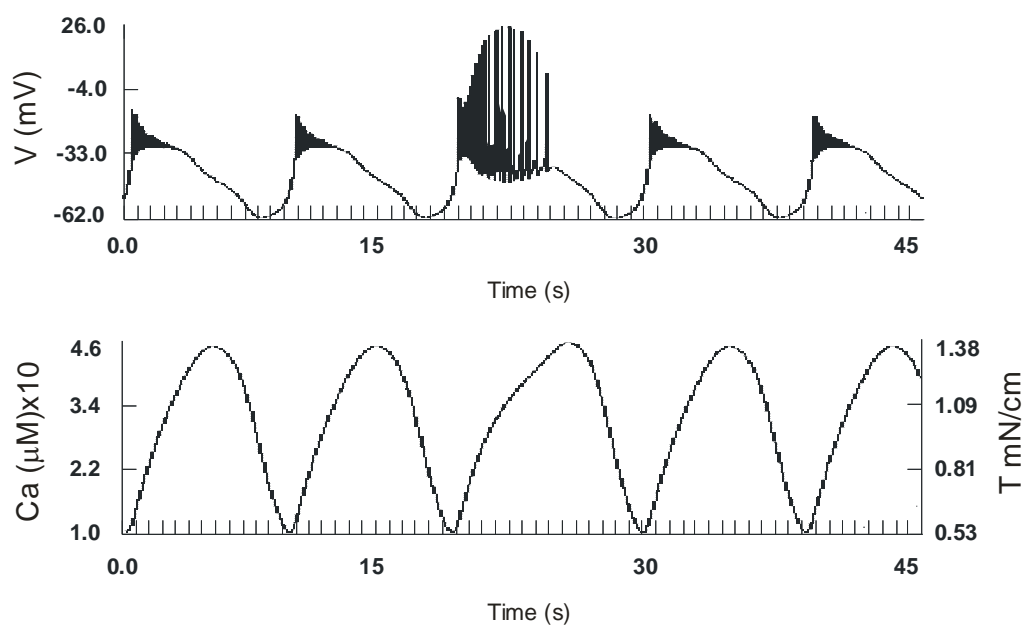

Figure 1: Electromechanical activity in the myofiber in response to a single stretch of the intensity $\varepsilon=0.85$ and duration $2 \mathrm{~s}$.

A gradual increase in the concentration of intracellular calcium ion leads to an increase in the permeability of $\mathrm{Ca}^{2+}$ channels. The rate of influx/outflux of $\mathrm{Ca}^{2+}$ ions is $0.02 \mu \mathrm{M} / \mathrm{sec}$. Maximum internal calcium concentration $0.46 \mu \mathrm{M}$ is achieved just before the slow wave reaches its resting value and each minimum is observed when the level of depolarization is near its maximum. This pattern of behavior changes with the appearance of the first irregular spikes followed by bursts. During this period, the concentration of internal calcium smoothly increases concomitantly with bursting and reaches its maximum just after the end of bursting. It continues to decrease to its minimal value until a new burst pattern begins and the process repeats.

Part of the free intracellular $\mathrm{Ca}^{2+}$ reaches the contractile protein system and, as a result, rhythmic contractions of the locus occur. The contractions are concomitant in phase and time with the dynamics of calcium oscillations. The contractions have an average amplitude $8.6 \mathrm{~g}$ and the calculated velocity of twitch is $1.72 \mathrm{~g} \cdot \mathrm{s}$. The maximum force generated by the gastric myofiber is $17 \mathrm{~g}$.

The myofiber demonstrates self-excitatory behaviour. As a response to the above changes in force, deformations of different amplitude are developed. Thus, a produced wave of deformation, $\varepsilon=0.3$, is sufficient to excite the mechanoreceptors. This level of depolarization is strong enough to excite the soma of the primary sensory neuron and to initiate the sequence of electromechanical events as described above. 

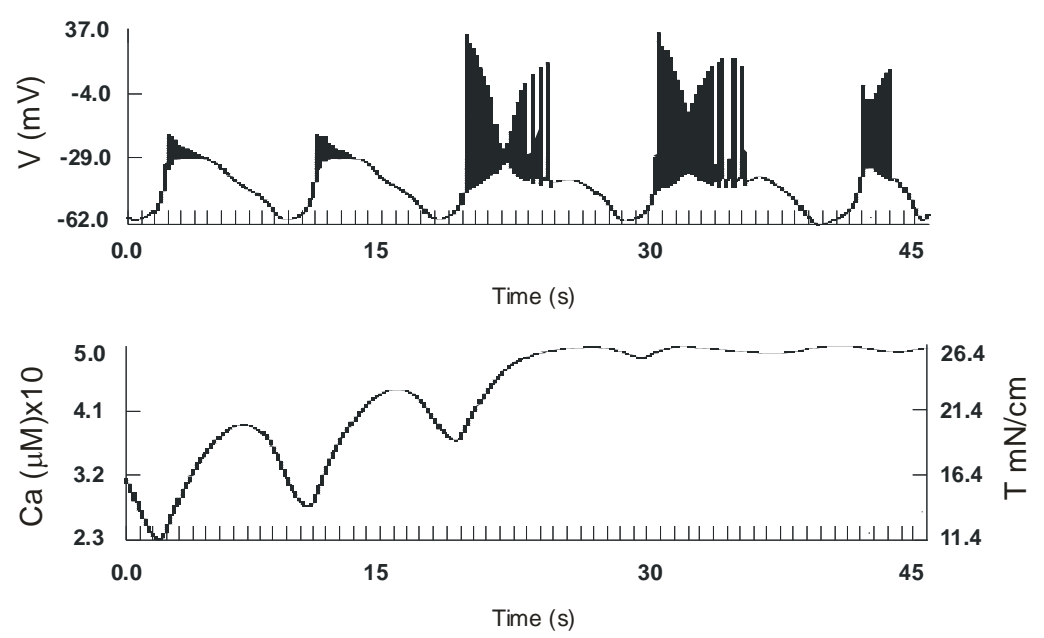

Figure 2: Electromechanical activity in the myofiber in response to a single stretch of the intensity $\varepsilon=0.5$ and duration 5 seconds.

An increase in the duration of stretching to $5 \mathrm{~s}$ is associated with a shift in the pattern of electrical activity to the regular busting type. The myofiber generates action potentials of amplitude $40 \mathrm{mV}$ at a frequency $5 \mathrm{~Hz}$. The high level of activation of L- and T-type calcium channels leads to a strong influx of $\mathrm{Ca}^{2+}$ ions. The maximum concentration of free cytosolic calcium reaches $0.45 \mu \mathrm{M}$. As a result, the myofiber undergoes a tonic-type contraction and generates the maximum force of $29.2 \mathrm{~g}$ (fig. 2).

\subsection{Responses to a periodic deformation}

Periodic stimulation of the myofiber with five stretch impulses $\varepsilon=0.85$ at a frequency $0.5 \mathrm{~Hz}$ causes multiple electrical responses in the afferent pathway. The characteristic dendritic action potentials of amplitude $82.5 \mathrm{mV}$ are generated at a frequency which coincides with the frequency of mechanoreceptor excitation. However, the primary sensory neuron responds only to the first three dendritic action potentials with three bursts of spikes of maximum amplitude $81.3 \mathrm{mV}$. It fails to respond to the fourth excitatory stimulus while the fourth discharge appears as a normal event and coincides with the fifth discharge at the mechanoreceptors. (The dynamics of the processes described below is similar to the dynamics of the electrical processes described in the previous section. Therefore, hereafter we shall only point out only qualitative differences of the process. Quantitative information will be provided where it differs from that in the analysis above). Three EPSPs of constant amplitude $(87.1 \mathrm{mV})$ are recorded from the soma of the secondary sensory neuron. As a result, the secondary neuron produces three bursts of high amplitude spikes, $102.1 \mathrm{mV}$, at a frequency $10 \mathrm{~Hz}$. The three resultant excitatory postsynaptic potentials activate the L-type 

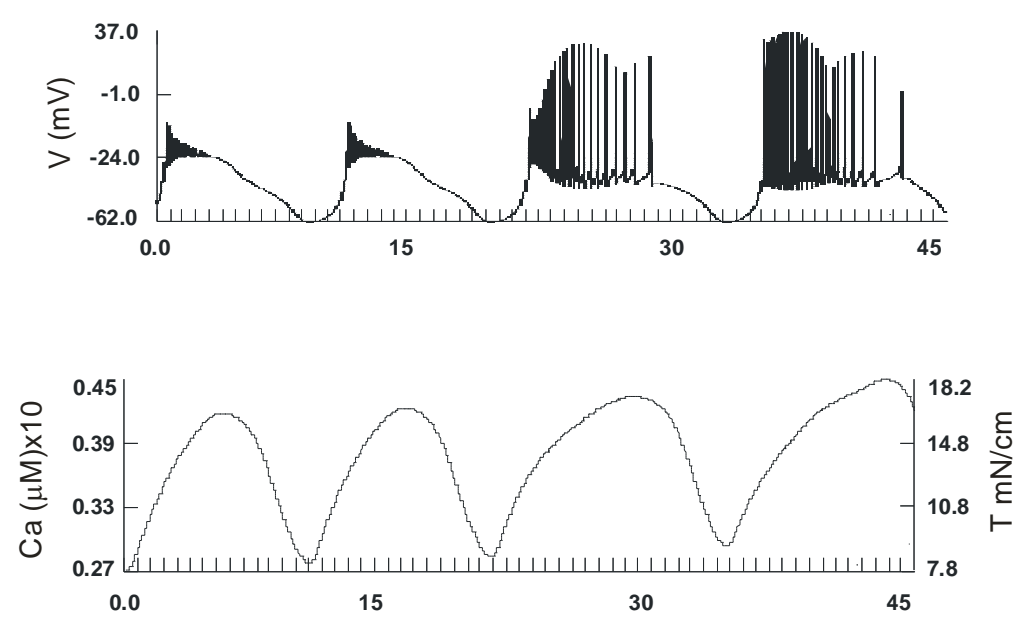

Figure 3: Electromechanical activity in the myofiber in response to a periodic stretch of the intensity $\varepsilon=0.85$ and frequency $0.5 \mathrm{~Hz}$.

$\mathrm{Ca}^{2+}$ channels to generate a long-lasting period of myoelectrical activity in the myofiber. During this period, repetitive bursts of maximum frequency are seen (fig. 3). The following short interval corresponds to irregular bursting discharges which are generated at a fluctuating frequency. The spikes produced maintain excitatory activity of the system, which again converts into a bursting mode of electrical activity and fires high amplitude spikes. The above changes increase the intracellular calcium ion influx and, as a result of the activation of the contractile protein system, more prolonged periodic contractions, with an active force of amplitude $18.2 \mathrm{~g}$, are produced.

\subsection{Responses to intermittent regime of deformation}

The stimulation of a gastric smooth muscle fiber with external impulses of deformation of variable intensity $(0.3 \leq \varepsilon \leq 0.8)$ demonstrates the intrinsic adaptive properties of the tissue. The free nerve endings respond to low frequency excitation with the generation of action potentials of maximum amplitude $82.5 \mathrm{mV}$. A simultaneous increase in frequency and decrease in amplitude of stretches distorts the shape of the action potential produced and reduces the amplitude of the spikes $50 \mathrm{mV}$. A further decrease in the rate of the stimulus and increase in its duration changes the pattern of electrical response to a prolonged depolarization at a constant level, $32 \mathrm{mV}$.

As a result, action potentials generated at the level of the free nerve endings, the soma of the primary sensory neuron discharges high-amplitude characteristic spikes of maximum amplitude, $81.3 \mathrm{mV}$. The number of EPSPs recorded at the soma of the secondary neuron is reduced to two and, as a result, only two longlasting discharges are seen. Their quantitative characteristics do not differ from those discussed in the previous paragraphs. 

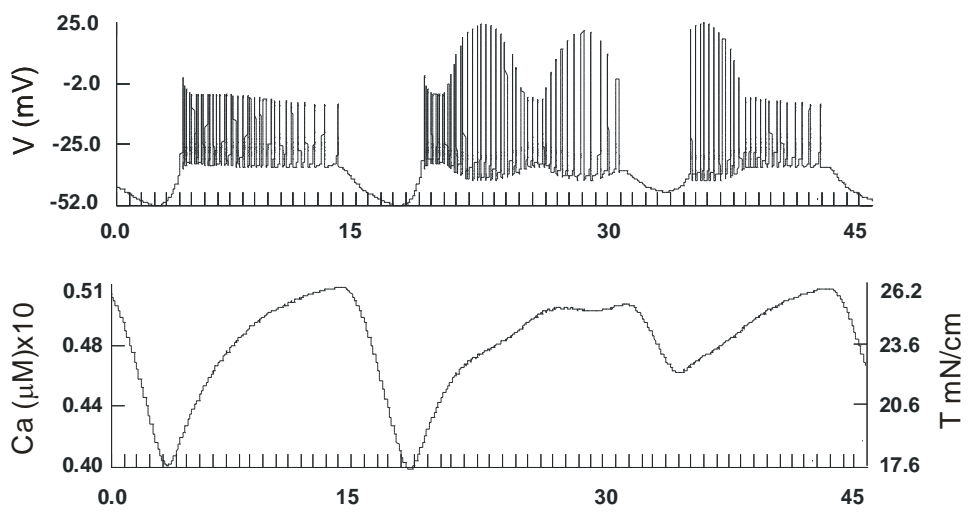

Figure 4: Electromechanical activity in the myofiber in response to an intermittent stretching.

The myoelectrical activity demonstrates cyclic changes in the pattern of discharges from slow wave electrical activity to bursting chaos followed by regular bursts (fig. 4). The periodic shift between regular bursting activity and irregular bursts is characteristic of the self-excitatory property of the smooth muscle syncytium. The frequency of recorded oscillations varies: $2.4-3.5 \mathrm{~Hz}$.

Calcium ion influx through the activated $\mathrm{Ca}^{2+}$ channels in the smooth muscle membrane results in a phasic tension response. The myofiber generates active force of the maximum amplitude, $0.51 \mathrm{~g}$, during the regular bursting mode of myoelectrical activity. Note that the intermittent regime of excitation does not provide sustained self-excitation of gastric muscle. The maximum contraction is sufficient to cause a single wave of deformation, $\varepsilon=0.32$, which is strong enough to stimulate the sensory elements of the afferent pathway with excitation of the fast calcium channels in the smooth muscle membrane. However, it fails to maintain a self-excitatory regime and it returns to an unexcited silent phase with the generation only of characteristic slow waves.

\section{Discussion}

Gastrointestinal motility reflects different kinds of processes, electrical, mechanical and chemical, linked together in complex ways. The behavioral nonlinearity, both of the separate neural and muscular compartments and of the integrated system they form to accomplish the motions of the stomach, makes it impossible to formulate a conceptualized general theory of motility from the use of reductionist methods. The integrative technique of mathematical modeling offers the possibility to solve this problem.

One of our prime goals in modeling was to develop a physiologically relevant and clinically applicable model of a myofiber of the stomach. The model proposed is based on electrophysiological data of smooth muscle cells. Our model assumption is that the activity of voltage-gated L-type $\mathrm{Ca}^{2+}$-channels is modulated by cholinergic neurons, which is consistent with physiological 
evidence. At present, because of lack of sufficient quantitative information about evolution of the channels, as a first approximation, we suppose that their activation is according to the step function.

Before we proceed further with the analysis of the numerical results, we must point out the following. Although we have simulated real conditions, i.e., the electromechanical response of a gastric myofiber to mechanical deformation of different intensities, our numerical results cannot be fully compared to published experimental data because it is technically impossible to record simultaneously in tissue the electrochemical and electromechanical processes from the neuronal elements and the smooth muscle. Only the integrative model approach adopted here allows us to display concurrently the complex sequence of events and to study their dynamic interactions. Therefore, we can only compare the behavior of separate structural elements of the system to existing experimental results.

The model is able to simulate accurately the depolarization, the transmembrane $\mathrm{Ca}^{2+}$ influx and the consequent contractions of the smooth muscle syncytium. The results of numerical simulation qualitatively and quantitatively agree with experimental observations. According to the obtained results slow waves generate an active force of the amplitude $8.6 \mathrm{~g}$. In our calculations, the dynamics of tension development is concomitant in phase and time with fluctuations of calcium.

The numerical results are consistent with results obtained experimentally from neurons of the submucous and myenteric nerve plexuses. The comparison of the theoretical results obtained with experimental data published in physiological media, i.e., patterns of discharges, the shape, amplitude, frequency and duration of spikes generated by the primary (sensory) and secondary (motor) neuron demonstrates qualitative and quantitative agreement $[10,11]$. The recordings of electrophysiological activity from the somas of neurons demonstrate that the amplitude of discharges varies between 75 and 85-90 (mV). Our numerical results of the firing activity of the primary and secondary neurons, respectively, are $81.3 \mathrm{mV}$ and $102.1 \mathrm{mV}$.

\section{Conclusion}

Numerical results of responses of the model to external stimuli demonstrate good quantitative and qualitative agreement with some experimental recordings presented in the literature. However, a detailed study and rigorous comparison, in a physiologically meaningful manner, of theoretical and experimental data is required for justification and proof of the robustness of the model.

\section{Acknowledgements}

We are grateful to Dr K. Tabbara and Dr K. Al Roomi for their support of the project. Mr Omar Al-Qabandi dedicates this work to his mother, Aisha Ali Jaffar. 


\section{References}

[1] Sanders K.M., Ward S.M. Interstitial cells of Cajal: a new perspective on smooth muscle function. Journal of Physiology 576.3, pp. 721-726, 2006.

[2] Forrest A.S., Ördög T., Sanders K.M. Neural regulation of slow-wave frequency in the murine gastric antrum. Journal of Physiology 290.3, pp. 486-495, 2006.

[3] Du P., Li S., O’Grady G., Cheng L.K., Pullan A.J., Chen J.D.Z. Effects of electrical stimulation on isolated rodent gastric smooth muscle cells evaluated via a joint computational simulation and experimental approach. Journal of Physiology 297.4, pp. 672-680, 2009.

[4] Edwards F.R., Hirst G.D.S. Mathematical description of regenerative potentials recorded from circular smooth muscle of guinea pig antrum. Journal of Physiology 285.4, pp. 661-670, 2003.

[5] Corrias A., Buist M.L. A quantitative model of gastric smooth muscle cellular activation. Annals of Biomedical Engineering 35.9, pp. 1595-607, 2007.

[6] Du P., O'Grady G., Windsor J.A., Cheng L.K., Pullan A.J. A tissue framework for simulating the effects of gastric electrical stimulation and in vivo validation. IEEE Transactions of Biomedical Engineering, 56.12, pp. 2755-2761, 2009.

[7] Corrias A., Buist M.L. Quantitative cellular description of gastric slow wave activity. American Journal of Physiology, Gastrointestinal and Liver Physiology, 294, pp. G989-G995, 2008.

[8] Miftahof R., Nam H.G. Mathematical Foundations and Biomechanics of the Digestive System, Cambridge University Press, UK 2010.

[9] Miftahof R.N., Nam H.G., Wingate D.L. Mathematical Modelling and Simulation in Enteric Neurobiology, World Scientific, 2009.

[10] Bornstein J.C., Furness J.B., Kunze W.A.A. Electrophysiological characterization of myenteric neurons: how do classification schemes relate? Journal of Autonomic Nervous System, 48, pp. 1-15, 1994.

[11] Smith T.K., Bornstein J.C., Furness J.B. Distension evoked ascending and descending reflexes in the circular muscle of guinea pig ileum: an intracellular study. Journal of Autonomic Nervous System, 29, pp. 203-213, 1990. 